\title{
Using PIC and PIC-MHD to investigate the occurrence of Fermi-1 acceleration in oblique astrophysical shocks
}

\author{
Allard Jan van Marle, ${ }^{a, *}$ Artem Bohdan, ${ }^{b}$ Alexandre Marcowith, ${ }^{a}$ Martin Pohl ${ }^{b}$ and \\ Paul Morris ${ }^{b}$ \\ ${ }^{a}$ Laboratoire Universe et Particules de Montpellier (LUPM), \\ Place Eugène Bataillon, 34090, Montpellier, France \\ ${ }^{b}$ Deutsches Elektronen-Synchrotron (DESY), \\ DE-15738 Zeuthen, Germany \\ E-mail: allard-jan.van-marle@umontpellier.fr, artem.bohdan@desy.de
}

\begin{abstract}
We use numerical simulations to investigate whether Fermi first order acceleration can occur in oblique shocks. This process involves repeated shock-crossings by non-thermal particles and requires a mechanism by which particles that have crossed the shock can be reflected back towards it. Using the particle-in-cell (PIC) method, we follow the formation of the shock and determine the fraction of the particles that gets reflected into the upstream medium. Then, with this result, we use a combined PIC-MHD method to model the large-scale structure of the plasma and the magnetic field surrounding the shock and find out whether the reflected particles can trigger the instabilities in the upstream magnetic field that are required to reflect the particles back toward the shock. We find that the feasibility of this process in oblique shocks depends strongly on the Alfvénic Mach number, with higher Mach shocks being more likely to trigger the diffusive shock acceleration process.
\end{abstract}

$37^{\text {th }}$ International Cosmic Ray Conference (ICRC 2021)

July 12th - 23rd, 2021

Online - Berlin, Germany

\footnotetext{
${ }^{*}$ Presenter
} 


\section{Introduction}

Cosmic rays are charged particles that are accelerated to relativistic speeds in astrophysical shocks. This acceleration process requires that the particles repeatedly cross the shock, picking up speed each time they get reflected back toward the shock by the local magnetic field, a process known as Fermi 1 acceleration, or diffusive shock acceleration (DSA) [1]. In order for the magnetic field to be able to reflect the particles, it needs to fluctuate, something that can be induced by the presence of the charged particles that are reflected by the shock. The presence of these particles and the local current that they create can trigger so-called non-resonant hybrid (NRH) streaming instability [2]. Because the NRH instability scales with the current, and therefore with the particle velocity, they can continue to reflect the particles, even as they accelerate. This allows the process to continue efficiently irrespective of the velocity of the particle.

The occurrence of streaming instability has been confirmed numerically for quasi-parallel shocks (i.e. the magnetic field is aligned with the the direction of motion of the upstream gas.) using a variety of methods [e.g. 3-5]. However, the situation becomes more complicated for those shocks that are oblique in nature. Numerical results obtained by [3-5] using a PIC-hybrid method showed no measurable DSA for shocks with angles between the flow and the magnetic field of $\theta_{B} \geq 55^{\circ}$. [6], using the combined PIC-MHD method [7], did find DSA for such oblique shocks. However, those models relied on an assumption as to how large a fraction of the particles was reflected at the shock. An assumption that was likely too high [8]. We below investigate this situation in detail, using a PIC code to obtain the non-thermal particle injection rate and a PICMHD code to model the shock large-scale structure and high Alfvénic Mach number regime. In all cases discussed below the upstream plasma beta parameter is set to one, hence the shock sonic and Alfvénic Mach numbers are of similar amplitude.

\section{Method}

For this numerical experiment, we need to combine two different methods. Firstly, we need to use the particle-in-cell (PIC) method to model the shock structure in order to determine the fraction of particles that gets reflected into the upstream medium. Once this has been established, we can use this injection rate to run further simulations with the PIC-magnetohydrodynamic (MHD) method [7] to determine whether the injection rate suffices to trigger the streaming instability.

PIC shock simulations are performed using an optimized fully-relativistic electromagnetic 2D PIC code with MPI-openMP hybrid parallelization developed from the TRISTAN code [10, 11]. Shocks are initialized using the reflecting-wall setup. Interaction of the upstream electron-ion plasma flow with the reflecting wall results in a shock which propagates towards the upstream plasma flow in positive $\mathrm{x}$ direction. The large-scale magnetic field, $\mathbf{B}_{\mathbf{0}}$, is carried by the upstream plasma. The shock obliquity angle, $\theta_{B n}$, is defined as the angle between the upstream magnetic field and the shock normal vector. For each obliquity angle we perform two simulations, with in-plane $\left(\varphi=0^{\circ}\right)$ and out-of-plane $\left(\varphi=90^{\circ}\right)$ magnetic field configuration, where $\varphi$ is the angle between the upstream magnetic field and y-axis which lies within the simulation plane. PIC simulation parameters are listed in Table 1. 
Table 1: PIC-MHD simulation Parameters

\begin{tabular}{lccccccr}
\hline \hline Name & $M_{\mathrm{A}}$ & $\theta_{B}$ & $V_{\text {sh }} / c$ & $N_{\text {inj }} / N_{0}$ & $U_{\text {inj }} / U_{\text {sh }}$ & $U_{\text {inj }} / U_{\mathrm{B}}$ & Result \\
\hline PIC01 & 20 & $65^{\circ}$ & 0.133 & $1.4 \times 10^{-6}$ & $2.9 \times 10^{-5}$ & 0.01 & - \\
PIC02 & 20 & $60^{\circ}$ & 0.133 & $3.7 \times 10^{-5}$ & $5.1 \times 10^{-4}$ & 0.2 & - \\
PIC03 & 20 & $55^{\circ}$ & 0.133 & $4.1 \times 10^{-4}$ & $4.1 \times 10^{-3}$ & 1.6 & - \\
PIC04 & 20 & $50^{\circ}$ & 0.133 & $2.7 \times 10^{-3}$ & $1.9 \times 10^{-2}$ & 7.6 & - \\
PIC05 & 20 & $45^{\circ}$ & 0.133 & 0.018 & 0.094 & 38 & - \\
\hline \hline PICMHD01A & 20 & $60^{\circ}$ & 0.1 & $1.0 \times 10^{-4}$ & $4.0 \times 10^{-4}$ & 0.2 & No significant DSA \\
PICMHD01B & 20 & $60^{\circ}$ & 0.05 & $1.0 \times 10^{-4}$ & $4.0 \times 10^{-4}$ & 0.2 & No significant DSA \\
PICMHD02 & 50 & $60^{\circ}$ & 0.05 & $1.0 \times 10^{-4}$ & $4.0 \times 10^{-4}$ & 1.25 & partial DSA \\
PICMHD03 & 100 & $60^{\circ}$ & 0.05 & $1.0 \times 10^{-4}$ & $4.0 \times 10^{-4}$ & 5 & partial DSA \\
PICMHD04 & 300 & $60^{\circ}$ & 0.05 & $1.0 \times 10^{-4}$ & $4.0 \times 10^{-4}$ & 45 & full DSA \\
\hline
\end{tabular}

$U_{\text {inj }} / U_{\mathrm{B}}$ is calculated assuming that $U_{\mathrm{inj}} / U_{\mathrm{sh}}=5.0 \times 10^{-4}$. PIC simulation are limited in time $\left(T_{\text {sim }} \approx 30 \Omega_{i}^{-1}\right)$, therefore production of the upstream turbulence is not possible.

For the PIC-MHD simulations, we use the same code described in [6], which is based on the MPI-AMRVAC code [9]. This code combines aspects of both traditional magnetohydrodynamics (MHD) and PIC in that it treats the thermal plasma as a fluid through MHD and the non-thermal charged particles through the PIC method. These two components interact with each other through the equation of motion for the particles and a modified version of Ohm's law for the fluid [7]. We set up our simulations in the rest-frame of the shock, starting from the analytical solution of the Rankine-Hugoniot conditions. Initially, there are no non-thermal particles in the grid, but, once the simulation has started, we begin to introduce the particles at the shock front according to the injection rate given in Table 1. This is done based on the injection rate found by the PIC simulations, but multiplied by a conservative factor two, the latter is required because the PIC results only count the particles reflected into the upstream medium, whereas the PIC-MHD method injects the particles isotropically in the restframe of the post-shock medium. N.B. A factor two is a conservative estimate and the actual injection rate is likely to be higher. However, the factor two guarantees that the number of particles moving upstream is similar in both simulations. PIC-MHD simulation parameters are listed in Table 1.

\section{PIC results}

At oblique shocks ions are injected towards the shock upstream after acceleration via few shocks drift acceleration (SDA) cycles [12]. During each SDA cycle most of participating ions (about 75\%) are advected downstream and the remaining part are accelerated by the motional electric field at the shock upstream. At high-obliquity shocks ions should go through larger number SDA cycles in order to achieve necessary escaping velocity (energy). Therefore the total injected energy drops at high-obliquity shocks, even though the average energy of injected ions is larger. 


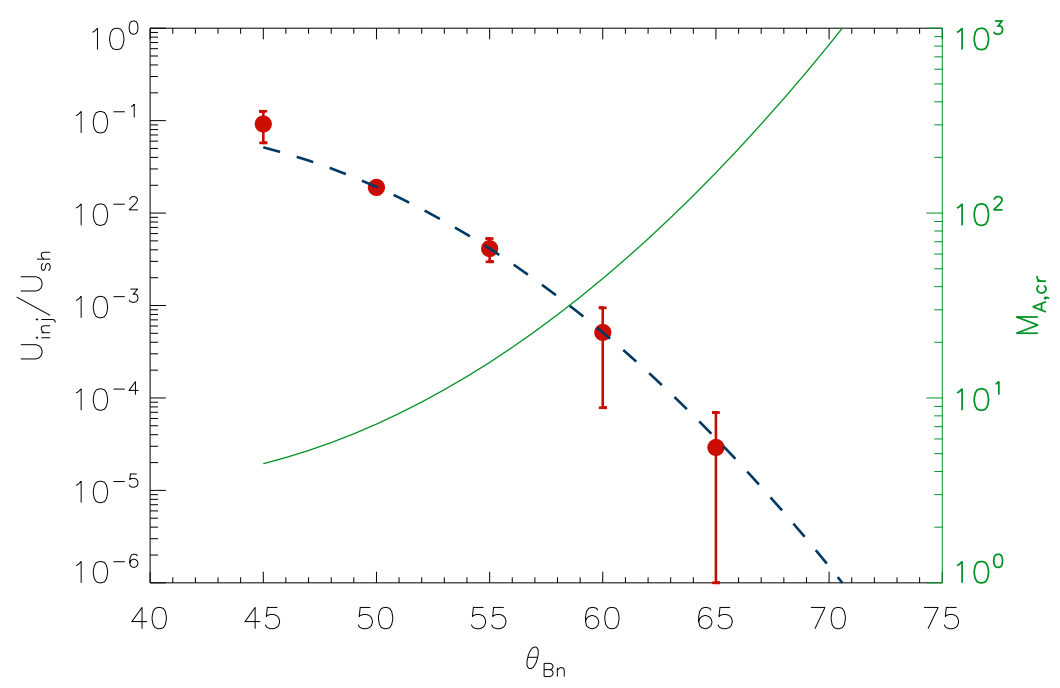

Figure 1: PIC simulations data (red dots), the best-fit to the PIC simulation data (blue dashed line) and the critical Mach number (green line).

The energy injection rate obtained by the PIC simulations as a function of the angle is shown in Fig. 1 and listed in Table 1. It is calculated as an average between in-plane and out-of-plane cases for selected $\theta_{B n}$. Also test simulations (not shown here) suggest that the ions injection rate does not depend significantly on Alfvénic Mach number of the shock and the upstream plasma beta. The critical Mach number indicates the Alfvénic Mach number at which the energy of the injected particles exceeds the energy of the upstream magnetic field and the non-resonant mode potentially can be excited.

AM: Artem could you add the Mach 30 run results ? to illustrate how does the injection rate varies with it. We need it to justify to keep the same injection rate in the PIC-MHD while exploring high Mach number values.

Artem: I would leave the current set of simulations, because for higher Mach number I have only out-of-plane simulation, while here I show averaged results for in-plane and outof-plane cases. I just add a sentence about weak (if any) influence of Mach number and the upstream plasma beta.

\section{PIC-MHD results}

Starting from the injection rate determined by the PIC simulations described in the previous section, we now use the PIC-MHD method to run a series of simulations of oblique shocks at Alfvénic Mach numbers ranging from 20 and 300. We keep the same injection rate irrespective of the Alfvén Mach number as this one does not show strong variation in PIC simulations when we vary $M_{\mathrm{a}}$ from 20 to 30 . We run the simulations in $2.5-\mathrm{D}$, using a box that is $480 \times 30 R_{\mathrm{g}}$, with $R_{\mathrm{g}}$ the gyro radius of the particles determined by the upstream magnetic field and the injection velocity, which we set to twice the shock velocity. Figure 2 (left panel) shows the gas density relative to the initial upstream density (bottom), the non-thermal particle density relative to the thermal gas density (center), and the magnetic field strength relative to the initial upstream magnetic field with 

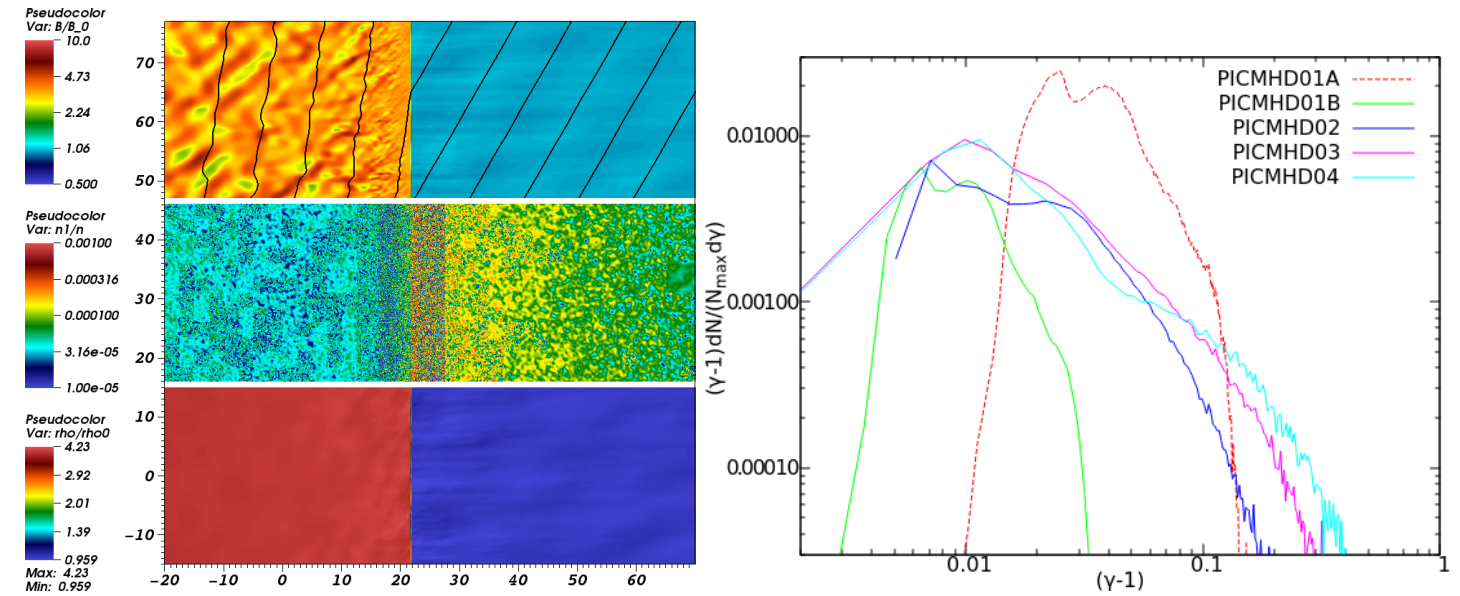

Figure 2: Left panel: The result for the PIC-MHD simulation with $M_{\mathrm{A}}=300$ at $t=300 R_{\mathrm{g}} / v_{\text {inj. }}$. From top to bottom, the magnetic field strength relative to the initial upstream magnetic field as well as the magnetic field lines, the non-thermal particle density relative to the thermal gas density, and the thermal gas density relative to the initial upstream gas density.

Right panel: SEDs for different Alfvénic Mach numbers. At $M_{\mathrm{A}} \geq 50$, the SED shows signs of DSA.

the magnetic field lines (top) for simulation PICMHD04 $\left(M_{\mathrm{A}}=300\right)$. Both the thermal gas density and the magnetic field show upstream and downstream variation, but the field lines upstream of the shock appear almost completely straight.

We show the resulting spectral energy distributions in Fig. 2 (right panel). At $M_{\mathrm{A}}=20$, we find very little evidence of DSA. The SED shows a double peak and then drops off quickly at higher energies. The low-energy peak represents the injection energy. The high-energy peak represents those particles that have been accelerated through SDA. The simulation with higher velocity (PICMHD01A) shows the same behaviour as the low velocity counterpart (PICMHD01B), indicating that the result does not depend on absolute velocity.

However, a high-energy tail starts to appear at $M_{\mathrm{A}} \geq 50$, extending further for higher Alfvénic Mach numbers. For $M_{\mathrm{A}}=50-100$, the SED still drops off quickly at higher energies, but at $M_{\mathrm{A}}=300$ we start to see the beginning of a plateau (between $(\gamma-1)=0.03-0.1$, indicating that DSA is becoming efficient, despite the small disturbance of the upstream magnetic field. This result is consistent with the results displayed in Fig. 1 where at an obliquity angle of $\theta_{\mathrm{Bn}}=60^{\circ}$ the critical Alfvénic Mach number is $\sim 50$.

\section{Conclusions}

Our results indicate that DSA is possible for oblique shocks. However, in order to trigger the required instabilities, the energy of the reflected particles needs to be at least equal to the magnetic field energy in the pre-shock medium. Therefore, it is easier achieved for shocks with a high Alfvénic Mach number. The dependence of the critical Alfvénic Mach number on the angle between the magnetic field and the flow indicates that DSA can be reasonably expected for oblique astrophysical shocks at $55-65^{\circ}$. For higher obliquity shocks, $M_{A} \gtrsim 1000$ is likely required. While astrophysical shocks with such Alfvénic Mach numbers do exist, they often have speeds not much 
below the speed of light, putting them outside the scope of our simulations, which apply only to non-relativistic flows. Objects like pulsar winds, GRBs, jets from compact objects can harbour sub-relativistic to relativistic shocks. In this regime, the shock can become superluminal, in which case, upstream motion of the particles becomes impossible. This means no streaming instability can occur, no matter.

\section{Acknowledgements}

This work is supported by the ANR-19-CE31-0014 GAMALO project. PIC numerical experiments were conducted on resources provided by the North-German Supercomputing Alliance (HLRN) under the project bbp00033 and using the Prometheus system at ACC Cyfronet AGH.

\section{References}

[1] Drury, L.O'.C., 1983, Rep. Prog. Phys., 46, 973

[2] Bell, A. R. 2004, MNRAS, 353, 550

[3] Caprioli, D., \& Spitkovsky, A. 2014a, ApJ, 783, 91

[4] Caprioli, D., \& Spitkovsky, A. 2014b, ApJ, 794, 46

[5] Caprioli, D., \& Spitkovsky, A. 2014c, ApJ, 794, 47

[6] van Marle, A. J., Casse, F., \& Marcowith, A. 2018, MNRAS, 473, 3394

[7] Bai, X-N., Caprioli, D., Sironi, L., \& Spitkovsky, A. 2015, ApJ, 809, 55

[8] Haggerty, C.C. \& Caprioli, D. 2019, ApJ, 887, 165

[9] van der Holst, B., Keppens, R., \& meliani, Z. 2008, CPC, 179, 617

[10] Buneman, O., Computer Space Plasma Physics: Simulation Techniques and Software Eds.: H. Matsumoto \& Y. Omura, Tokyo: Terra Scientific, 1993

[11] Niemiec, J., Pohl, M., Stroman, T., et al. 2008, ApJ, 684, 1174. doi:10.1086/590054

[12] Caprioli, D., Pop, A.-R., \& Spitkovsky, A. 2015, ApJL, 798, L28. doi:10.1088/2041$8205 / 798 / 2 / \mathrm{L} 28$ 